\title{
HYGIENIC MYCOLOGY IN HUNGARY
}

\author{
E. K. NOvÁK and JUDIT ZALA
}

REAQUA Ltd., Budapest and Department of Mycology, “Johan Béla” National Center for Epidemiology,
Budapest, Hungary

In Hungary the medical mycological research concerning the systemic (so-called deep) mycoses started in the National Institute of Hygiene. The Mycology Department has been founded in 1955 in this Institute with nation-wide authority as a routine diagnostic laboratory for clinical specimens from systemic mycotic infections. Three years later its activity was broadened - on ground of the experiences - to cover the total field of hygiene, thus we had to establish the "mycologia hygienica" (hygienic mycology), however, this has not been accepted as an independent discipline. Here a short, selected overview is given of our experiences.

In the following the trends of the activity is demonstrated along the subdivisions of hygiene (epidemiology, environmental-hygiene, professional and occupational-, nutritional and food hygiene). In respect of hygiene, however, the fungi play a Janus-faced (more exactly Dr. Jekyll-Mr. Hyde) role, as they can behave not only harmful but also beneficial.

\section{Epidemiology}

Fungi may induce fungal lesions and diseases. Among them are allergies caused by the more than $100 \mathrm{spp}$. allergenic fungi. Both alimentary and respiratory allergies are frequent in the Hungarian population, especially in children (the bronchial asthma will be discussed later in environment hygiene). Monitoring of the patients with qualitative and quantitative IgE test (RAST and PRIST techniques) were done. Alternaria spp. and Cladosporium spp. proved to be the most frequent allergenic molds. Out of the previous the $A$. alternata species, however, showed allergen relationship to an other black mold Phoma betae.

The problematic of toxicoses (mycetismus; as well as the mold mycotoxicoses) caused by the more than $300 \mathrm{spp}$. of toxinogenic fungi belong to Alimentation and Food Hygiene (see below).

ERVIN K. NOVÁK

REAQUA Ltd.

Fehérvári út 144, H-1116 Budapest, Hungary

JUDIT ZALA

Department of Mycology, "Johan Béla" National Center for Epidemiology

P.O. Box 64, H-1966 Budapest, Hungary 
The most important human fungal harms are the tissue invasions caused by the more than $200 \mathrm{spp}$. of invasive fungi (dermato-mycological problems will be touched also in an other review). Clinical specimens from suspected "deep" mycosis or colonization (with opportunistic pathogens) were studied not only with classic laboratory diagnostic and identification techniques (direct microscopy of smears and tissue sections stained with various methods, as well as cultivation on general, selecting and differentiating media), but also with modern serologic-immunologic methods (Radial Immunodiffusion, Counter Immunoelectrophoresis, latex-agglutination, PRIST, RAST, ELISA), while new methods were also developed and introduced (India Ink Immuno Reaction and Enzyme Linked Immuno Sorbent Stain).

In the spectrum of the pathogenic- and colonizing agents (opportunistic pathogens) the prevalence of yeasts and yeast-like fungi was observed and Candida albicans proved to be the most frequent although Cryptococcus neoformans and Saccharomyces cerevisiae were also found in some instances.

Molds and dimorphic fungi, however, were much rare but some interesting cases were also found: Emericella nidulans (Aspergillus nidulellus sexual reproduction form) in a postoperative lung infection (first report in the literature on a cleistothecial aspergillome), Acremonium strictum meningitis, Coccidioides immitis arthritis, Histoplasma capsulatum generalized infections.

Dermatophytes. This group and the fungal skin diseases were not dealt with in the basic routine studies of the Department. However, some restricted data were collected (e.g. screening on foot dermatophytoses and dermal yeast infections). Last but not least we succeeded to induce budding of Trichophyton mentagrophytes in mouse peritoneum after cystein-cultivation, thus simulating the morphogenesis of true dimorphs (and other soil inhabitant geophilic Gymnoascaceae pathogens).

The source of infection is one of the most important factor in epidemiology. In addition to diseased and/or carrier humans and/or animals soil is a real source of infection as it serves as a biotope for many (geophilic) fungal pathogens, contradicting the classic theses of epidemiology (based on bacteriology and virology) which negate the role of soil as being a source of pathogens. Beyond the dimorphic fungi and obligate and potential pathogenic molds, dermatophytes are also inhabitants of soil and it succeeded to isolate from Hungarian - and Vietnam - soils all the worldwide known species of geophilic dermatophytes, moreover many in the teleomorphic (sexually reproductive) state. While the most frequent pathogenic yeast Candida albicans was found to survive outside the host only for a restricted period, it cannot be regarded as a real soil inhabitant and at worst may persist in soil within pathologic materials.

Host susceptibility and resistance have also a major role in fungal diseases. Natural (hereditary) susceptibility: only the observed definite nephrotropism and hepatotropism in experimental mice candidiosis is mentioned here. However, regarding human pregnancy, in contrast to the earlier epidemiologic and gynecologic belief the highest incidence of vaginal colonization gets not to the time of parthus but to the second trimester of pregnancy. Nosocomial (acquired) susceptibility is well illustrated e.g. by the significance of oral thrush in newborns and infants. Puerperal mothers' antibacterial, antibiotic and/or iron (Fe) treatment significantly increased the frequency of $C$. albicans mouth colonization and thrush in their newborn. 
A new form of "kidney-therapy", the Continuous Ambulant Peritoneal Dialysis (CAPD), gives also an opportunity for nosocomial fungal infections: peritonitis with various mold and yeast ethiology (only in 198915 Aspergillus niger cases were diagnosed). Sometimes prior of use the fresh dialyzing fluids were found to be contaminated by fungi (because of visible and invisible damages of the sack).

Growth of the pathogen. In the "antibiotic era" an increased incidence of systemic mycoses were observed in the treated patients, thus a direct growth promoting effect of the antibiotics on pathogenic fungi was supposed, moreover observed in (inadequate) experiments. With adequate studies no in vitro growth stimulation could be confirmed. While in vivo, in experimental candidosis in mice, some antibiotics (chloramphenicol, erythromycin and rovamycin) proved to be aggravating, also with an induction of earlier hematogeneous dissemination. Some others, however, showed a pseudo-curative (pseudotherapeutic) effect (anti-tuberculotics: streptomycin, viomycin, and INH). Explanation of this protection lays in the induction of intraperitoneal coalescence at the site of administration (thus fixing the pathogen and help to the defense). Other tuberculostatics: pyrazin amide, cycloserin, and p-amino salicylic acid proved to be indifferent.

Referring to antifungal substances both efficiency and mode of action were studied. Out of the preservatives used in foodstuffs and pharmaceutics sorbic acid could only temporally inhibit some of the studied yeasts (C. alb., C. claussenii, C. kefyr, $C$. tropicalis and C. utilis), because of the aerobic degradation (and even assimilation) of it by these species. While the sometimes weak antifungal effect of p-oxybensoic acid methyl- and propyl esters (Nipagin and Nipasol) was due to their protein binding. From disinfectants a comparison was made of 14 commercial products in anti-C. alb. efficiency (killing 99\% of the cells within 2 min.). The weak, if any, effect of the hexachlorophen containing compositions, Ritosept and Solutio hexachloropheni, originated from their alkali or ethanol content, respectively. A cleansing combination type the "lysoform" (liquor formaldehydi saponatus; containing also ethanol, and potassium-soap) leaving an assimilable residue on the disinfected object makes it with time invadeable for fungi. An easy termination of the effect of the cetyl pyridinium bromide containing preparations (Nitrogenol and Sterogenol) was found in their inactivation by zinc ions. By the combination of low (0.2 M, non-fungicidal and less corrosive) concentration of hydrogen peroxide with low (slightly fungicidic and less toxic) concentration of formaldehyde an efficient disinfectant was achieved. It is regrettable that the best antifungal preservation and disinfectant compound pentachlorophenol had to be disclosed because of its high mammalian toxicity. However, it was earlier used with the best efficiency against molding of painted or paper covered wall surfaces and floor carpets. For the last aim only Eulan Asept P was also a convenient impregnation compound. While PCP was tried to be introduced into disinfecting wash-detergent combinations, its sensitivity to the type of the detergent compound and other additives (viz. emulsifiers) could be observed.

With antifungal chemotherapeutics and polyene antibiotics the efficiency (i.e. the susceptibility against these medicines of the isolated pathogens) are regularly checked because of the natural and induced resistance of the fungal strains. Because of the constitutive and acquired resistance of the pathogenic fungi towards the antifungal drugs (azole derivatives and polyenic antibiotics) the study of the mechanism of action of the later is not irrelevant. Regarding the antifungal antibiotics - the polyenes - in contrast to 
the widespread belief on their common mechanism of action: i.e. complexing with ergosterol and inducing transverse physical channels in the plasma membrane of the fungal cells leading to loss of $\mathrm{K}^{+}$by passive efflux etc., a more complicated mechanism of action was rendered probable based on the studies of ion movements, amino acid transport and comparison with real membrane damaging agents.

Beneficial roles of fungi in medicine are played in their direct and indirect (recombinant DNA techniques) biotechnological utilization as producers of antibiotics, vitamins, and hormones. In this field for supporting the checking of the first two phases (physical fusion of membranes, and plasmas) in the protoplast fusion technique an IndiaInk Immune Reaction and an Enzyme Linked Immuno Sorbent Stain was developed.

\section{Environmental hygiene}

Fungal contamination of soil, surface waters, air, inside surface of flat border walls (panel dwelling estates), stored raw materials, intermediary and end products, objects, etc., was extensively studied. In air-hygiene the fungal pollution over 500 particles $/ \mathrm{m}^{3}$ is considered to be a physical overload of the lungs in addition to the danger of invasive, allergenic and toxic effects. Outdoor air pollution was monitored not only in Budapest, but also in agricultural areas, inside and outside villages. Beside molds yeasts were also registered. Among indoor studies the air of hospital boards, nursery, microbial laboratory and many metropolitan flats in merely panel housing estates, were repeatedly sampled. Within the species spectrum considerably high participation (ca. 50\%) of the black molds could be observed, however they are more allergenic.

In water hygiene the fungal pollution of fresh water (small streams, rivers and the lake Balaton) were repeatedly investigated for the presence of molds and yeast species. Twenty-seven human pathogen species (while no dermatophytes) were found. It seems that 68 species of 20 genera were hitherto not found in Hungary, while 59 species of 20 genera were not mentioned from surface waters by the most comprehensive US project.

In some cases, however, sewage plants were also studied at recreation areas. In effluents - besides the considerable decrease in population density - C. famata and Sacch. cerev. were found to be the most frequent. C. albicans, however, failed to appear although excrement was abundant in the sewage. Other pathogenic yeasts were also abundant in the influents.

Beneficial role of fungi in respect of environmental hygiene is realized in the decomposition (metabolization, humification) of environment-polluting plastics, sewage, styrene, phenol and formaldehyde by various fungus species. However, the dangerous level of nitrate and/or ammonia could be also eliminated by fungi. Fungal detoxification is an other route of elimination of various toxic or poisonous substances (e.g. pesticides) resulting in atoxic metabolites (e.g. glutaminic acid conjugates). For heavy metals fungal absorption - more exactly accumulation (biofiltration) - comes also into consideration in environmental protection. By adaptation considerably increased tolerance could be achieved with various molds and yeast species, however in the literature extreme value was published with Trichosporon cerebriforme $\left(28 \% \mathrm{CuSO}_{4}\right.$ in $\left.1.0 \mathrm{H}_{2} \mathrm{SO}_{4}\right)$. 


\section{Professional and occupational hygiene}

Toxic, allergic, invasive injuries through air pollution and/or contact with fungi, may be acquired during occupation. We introduced to distinguish Primary fungal Industries, Factories and occupations (fungi and/or its products are participants or aim of the technology; positive role) such as Biotechnology; food industry or staff of mycologic laboratories; and Secondary fungal ones (fungi are undesirable invaders of the technology; negative role): e.g. non-bio-industries and employment: paper-mills, cellulose factories, tanneries, archeology-museology etc. However, in bio-industry and agriculture the fungal contamination of raw-materials, intermediary and finished products is also frequent. Thus it is very difficult (and expensive) to prevent air-pollution in primary type factories, while very easy in secondary types. The fungal damages and diseases acquired in the primary fungal factories and occupations fall into the group of the specific professional damages and diseases, while those acquired in secondary fungal factories and occupations belong to the non-specific professional damages and diseases. The highest Fungal Air Pollution in numerous primary fungal industries and occupations was found with "blue cheese" mold inoculum cultivation at conidium harvesting $\left(46 \times 10^{6} \mathrm{cfu} / \mathrm{m}^{3}\right.$ air). An interesting fungal air-pollution in secondary fungal industries and occupations was observed in a localization tower (with inside plastic covered walls and boric acid solution molded by Asp. fumigatus) in a reactor block of an Energy Enterprise (electric power works) in Trans-Danubium, where the workers were monitored for fungal allergy.

As it was suspected "soaked skin" especially in food-industry proved to be a predisposing factor for dermal yeast infections of the workers' hand, while for "athlete's foot" perspiration was responsible.

\section{Nutritional and food hygiene}

Damages of agricultural raw and preprocessed materials are manifested in deterioration of foodstuffs caused by molds.

Out of the food preservatives sorbic acid (see in Epidemiology, antifungal preservatives) and pimaricin (polyenic antifungal antibiotic) were studied. The last one was found to induce giant cell production in C. albicans.

Beside the well-known deteriorations it seems worthy to mention an uncommon type of fungal foodstuff degradation. In a vinegar factory a fungal invasion by Monascus ruber and Moniliella acetabutans was capable of reducing the acetic acid production by assimilating it.

Beneficial effects are not only restricted to the well-known fermented drinks (alcoholic and non-alcoholic beverages) of western, African and far-east types, but fermented foods and foodstuffs are also worldwide involved (cf: Far East: tempeh, shoyu, etc.). Out of soft alcoholic beverages tea-fungus (komboucha) and Japan crystal both are symbiotic consortium of yeasts and acetic acid bacterium. The latter Japan fungus (white alga) was found to be white, amorphous, hard gel grains of ca. 4-6 $\mathrm{mm}$, grown on sucrose solution supplemented with 1-2 raisins, and was "consumed" as an universal medicine. It 
is, however, a symbiosis of Sacch. cerev. race prostoserdovi or Sacch. cerev. race uvarum, and Gluconobacter oxydans.

[The scientific stuff (long staying visitors also) since 1955, in chronological order: Anna Csillag (head: 1955-57), E. K. Novák (head: 1957-92), Györgyi Vörös-Felkai, Cs. Dobolyi, J. Galgóczy, T. Deák, Anna Pólay, L. Sztankov, L. Kustán, Judit Zala (head: 1992-), Nguyen Ngoc Thuy, Zsuzsa Horváth, J. Bitskey, Eman Hussien Ashour, T. Nagy; while many other colleagues in clinics and hygienic services also helped us.] 